\title{
Tracking Simulation of J-PARC Slow Extraction
}

\author{
K.A. Brown \\ Brookhaven National Laboratory
}

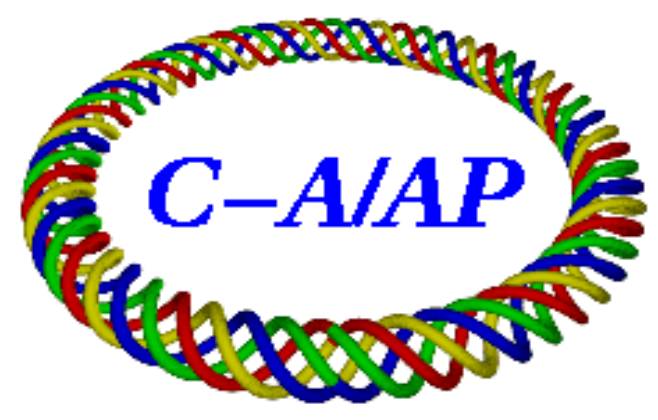

Collider-Accelerator Department Brookhaven National Laboratory Upton, NY 11973 


\title{
Tracking Simulation of J-PARC Slow Extraction
}

\author{
K.A. Brown, \\ Brookhaven National Laboratory, Upton, New York 11973, USA \\ March 12, 2007
}




\section{Abstract}

This report will describe tracking simulations of the J-PARC slow extraction system that were performed using a simulation program written for slow extraction from the AGS and AGS Booster. To adapt this simulation program to J-PARC was relatively simple, since the primary elements for tracking particles through driven resonances is an integral part of the simulator. The simulation works in 5 dimensions (two transverse plus the momentum coordinate), but does not include coupling except through chromaticity and through the perturbation of the resonances themselves.

The J-PARC slow extraction system is a unique design, with a lattice that has a negative $\gamma_{t r}$ and both horizontal and vertical chromaticities defined to be zero (or very near zero). The dispersion at the electrostatic septum is defined to be zero and the electrostatic septa are located to the inside of the accelerator aperture. The horizontal betatron tune is set to be below the tune of the resonance, requiring the horizontal tune to be ramped more positive during the extraction process. In combination with the near zero chromaticity this has the effect of extracting large amplitude particles first and then slowly peeling the beam in phase space until small amplitude particles are extracted last. In order to minimize the extracted beam emittance a dynamic bump is employed, which keeps the angular distribution of the extracted particles minimized. This will also improve the extraction efficiency, in that losses on downstream septa are minimized due to the rotation of the extracted phase space and the alignment of the septa. 


\section{Contents}

$\begin{array}{lc}\text { CONTENTS } & 3\end{array}$

$\begin{array}{ll}\text { LIST OF TABLES } & 3\end{array}$

$\begin{array}{ll}\text { LIST OF FIGURES } & 3\end{array}$

1 INTRODUCTION $\quad 4$

2 COMPUTER SIMULATION OF CHARGED PARTICLE BEAMS: A TUTORIAL 4

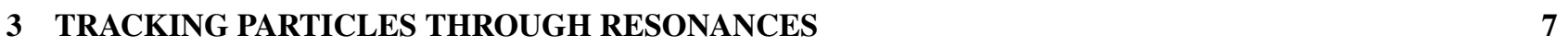

4 INCLUDING SEPTA AND MEASURING LOSSES 9

5 CERN MAD8 SIMULATION OF J-PARC EXTRACTION LATTICE 10

6 DYNAMIC BUMP FOR J-PARC SLOW EXTRACTION 11

7 TRACKING SIMULATION OF J-PARC SLOW EXTRACTION 12

8 AN ALTERNATIVE EXTRACTION SCHEME NOT REQUIRING DYNAMIC BUMPS 13

9 CONCLUSIONS $\quad 13$

10 ACKNOWLEDGEMENTS 14

11 REFERENCES 14

\section{List of Tables}

1 J-PARC Tunes and Chromaticities with and without a bump at ESS1 . . . . . . . . . . . . 10

2 Courant-Synder parameters used in tracking simulations. . . . . . . . . . . . . . . . . . . 10

3 Dispersion and orbit deformations. . . . . . . . . . . . . . . . . . . . 10

\section{List of Figures}

1 Gaussian generated phase space using uniform random numbers . . . . . . . . . . . . . . 6

2 Four particles passing into a sextupole resonance at the Booster D3 thin septum . . . . . . . . . . 8

3 Distribution of particles in $\mathrm{x}$ and $\mathrm{x}$ ' for idealized phase space. . . . . . . . . . . . . . . . 9

4 Change in orbit position and angle in extraction region for dynamic bump with constant position and angle that varies from 0 to $-0.25 \mathrm{mrad} \ldots \ldots \ldots \ldots \ldots$

5 Phase space at J-PARC ESS for 10 different particles (in black), including a dynamic bump. Step size in this example is $2 \mathrm{~cm}$ for small amplitude particles. $>990$ Particles in red have stepped past the septum (blue line at $-0.06 \mathrm{~m}$ ) out of 1000 initial particles. In this case the initial beam emittance is $2 \pi \mathrm{mm}$ mrad. The septum thickness is taken to be $0.051 \mathrm{~mm}(2 / 1000$ of an inch). Extracted particle phase space trajectories are also shown at the exit of ESS1 . . . . . . . . . . . . . . . . . . . . 12

6 The same conditions as in Fig. 5, except in this case the initial beam emittance is $6 \pi$ mm-mrad. . . . . . 13 


\section{INTRODUCTION}

Extraction of particles from accelerators using non-linear resonances can only best be analyzed using particle tracking simulations. Unfortunately there are few such simulators that can be adapted for use by accelerator physicists. This is particularly true when you want to simulate as close as possible the machine that exists or is being built. Fortunately it is not difficult to write such a simulation. The equations of motion are well understood and the techniques for tracking particles are well developed.

This report will give a detailed description of a simulator which was written to generate distributions of particles and study the passage of particles through excited resonances, such as those created to perform resonant extraction. As input the simulator uses Courant-Snyder parameters derived in some other manner (e.g., from MAD) and makes small adiabatic variations in those parameters in order to simulate passage through a resonance.

I should say that programs exist already that could be used to study slow extraction. There are a number of reasons for writing our own program. One reason is to give ourselves the most amount of flexibility and not be constrained by the assumptions that other programs may have on what is or isn't useful. Other programs were written for the purpose of solving certain classes of problems. But the main reasons for writing our own program are two; computational speed and the ability to simulation the parameter variations that are a natural part of slow extraction. No claim is made that this program is better in any way from others, nor that it is correct, until a sufficient degree of verification is performed. The program does not include corrections for space charge effects (e.g., tune shift during extraction), instabilities due to the impedance seen by the beam due to the geometry of the vacuum enclosure and materials, instabilities due to interactions with residual gas or electron clouds effects.

\section{COMPUTER SIMULATION OF CHARGED PARTICLE BEAMS: A TUTORIAL}

The motion of charged particles in linear systems of magnets can be described as a simple harmonic oscillator with the form: (Most of what appears in this section can be found described in much greater detail in [1].)

$$
\frac{d^{2} \eta}{d \phi^{2}}+\nu^{2} \eta=0
$$

where $\nu$ is the betatron tune and $\phi$ is the phase at a given point in the system. The general solution to this equation is,

$$
\eta(\phi)=A \cos (\nu \phi+\delta)
$$

where $\mathrm{A}$ and $\delta$ are constants of integration which depend on the initial conditions. The motion of this simple harmonic oscillator can be visualized by thinking of the system as a periodic system in equilibrium. The motion can then be described as a circle in $\left(\eta, \eta^{\prime}\right)$ space, where $\eta^{\prime} \equiv d \eta / d \phi$. In this case,

$$
\eta^{\prime}(\phi)=-A \sin (\nu \phi+\delta)
$$

To do tracking simulations of single particles through arbitrary linear systems we generate a distribution of coordinates using random numbers,

$$
\eta=\sigma_{x} \sqrt{-2 \ln \left(i_{1}\right)} \cos \left(2 \pi i_{2}\right)
$$

and,

$$
\eta^{\prime}=-\sigma_{x} \sqrt{-2 \ln \left(i_{1}\right)} \sin \left(2 \pi i_{2}\right)
$$

where $i_{1}$ and $i_{2}$ are two independent random numbers between 0 and 1 .

The distribution of coordinates will then describe a Gaussian distribution of particles with a one sigma width of $\sigma_{x}$. We now need to make a transformation from $\left(\eta, \eta^{\prime}\right)$ to coordinates useful for tracking the generated particles through a secondary system. So far the motion described is only for that of a particle in a single plane. To describe a distribution of particles in the two transverse planes (vertical and horizontal) we assume no coupling and use an independent set of random numbers to generate an independent distribution of coordinates.

The nominal coordinate system used in accelerators is a curve-linear coordinate system. In this case the trajectory is along a planar closed curve. The path length along the curve of motion is the independent variable, $\mathrm{s}$. At any point along this curve we can define three unit vectors: $\hat{s}, \hat{x}, \hat{x^{\prime}}$. A more detailed description of this coordinate system can be found in the MAD manual [2].

We generally are interested in deviations of $x$ and $y$ from the reference orbit, so in our discussion we speak of $x$ and $y$, meaning the deviations in horizontal and vertical planes from the reference path defined by the elements of the system.

In the curve-linear coordinate system, then, we write the equation of motion in the form of Hill's equation (note that $\left.x^{\prime} \equiv d x / d s\right)$,

$$
x^{\prime \prime}+K(s) x=0
$$


$\mathrm{K}(\mathrm{s})$ is a periodic function of the independent variable, $\mathrm{s}$. The general solution to this equation is

$$
x=A \omega(s) \cos (\psi(s)+\delta)
$$

Again, A and $\delta$ are constants of integration reflecting the initial conditions. By substituting $\omega(s)$ and $\psi(s)$ into eq. (6) we find

$$
2 \omega \omega^{\prime} \psi^{\prime}+\omega^{2} \psi^{\prime \prime}=\left(\omega^{2} \psi^{\prime}\right)^{\prime}=0
$$

Since what we want is a way of describing the motion of particles as they propagate through the system from some point $s_{0}$ to $s_{0}+C$, we re-normalize the constants to derive the Courant-Snyder parameters,

$$
\begin{gathered}
\beta(s)=\frac{\omega(s)^{2}}{k} \\
\alpha(s)=-\frac{1}{2} \frac{d \beta(s)}{d s} \\
\gamma=\frac{1+\alpha^{2}}{\beta}
\end{gathered}
$$

Now we rewrite the equation of motion to describe a mapping from one point in the reference trajectory to another point in the reference trajectory:

$$
\left(\begin{array}{c}
x \\
x^{\prime}
\end{array}\right)_{s_{0}+C}=M\left(\begin{array}{c}
x \\
x^{\prime}
\end{array}\right)_{s_{0}}
$$

where,

$$
M=I \cos \Delta \psi_{c}+J \sin \Delta \psi_{c}
$$

where we have defined

$$
I=\left(\begin{array}{ll}
1 & 0 \\
0 & 1
\end{array}\right), \quad J=\left(\begin{array}{cc}
\alpha & \beta \\
-\gamma & -\alpha
\end{array}\right), \quad J^{2}=-I
$$

I and $\mathrm{J}$ satisfy the symplectic condition, $\mathcal{M}^{T} S \mathcal{M}=S$. One consequence of this condition is the transformations are very close to exact. If a matrix $\mathcal{M}$ is symplectic, then its inverse is symplectic and the determinant of that matrix is symplectic. If two matrices $\mathcal{M}$ and $\mathcal{N}$ are symplectic, then the product $\mathcal{M N}$ is also symplectic. The only loss in precision will be due to round off errors resulting from finite bit sizes in numeric representations.

The constant $\mathrm{A}$ in eq. (7) can be expressed in terms of $x$ and $x^{\prime}$.

$$
\alpha(s) x(s)+\beta(s) x^{\prime}(s)=-A \sqrt{\beta(s)} \sin (\psi(s)+\delta)
$$

or,

$$
A^{2}=\gamma(s) x(s)^{2}+2 \alpha(s) x(s) x^{\prime}(s)+\beta(s) x^{\prime}(s)^{2}
$$

This invariant form describes an ellipse in $\left(x, x^{\prime}\right)$ phase space. The area of the ellipse is a constant of the motion and is

$$
\text { Area }=\frac{\pi A^{2}}{\sqrt{\beta \gamma-\alpha^{2}}}=\pi A^{2}
$$

The constant $A^{2}$ is called the unnormalized emittance, $\epsilon$, in which case,

$$
\frac{\epsilon}{\pi}=\gamma x^{2}+2 \alpha x x^{\prime}+\beta x^{\prime 2}
$$

The canonical transformations between $\left(\eta, \eta^{\prime}\right)$ coordinates and $\left(x, x^{\prime}\right)$ coordinates is then a Floquet transformation,

$$
\begin{gathered}
\eta=\frac{x}{\sqrt{\beta}} \\
\eta^{\prime}=\frac{x \alpha}{\sqrt{\beta}}+x^{\prime} \sqrt{\beta} \\
x=\eta \sqrt{\beta}
\end{gathered}
$$




$$
x^{\prime}=\frac{\eta^{\prime}-\eta \alpha}{\sqrt{\beta}}
$$

The computer code takes eqs.[4,5] and makes the canonical transformation to (x, $\left.\mathrm{x}^{\prime}\right)$, generating a single set of coordinates. The distribution of these coordinates depends on the value of $\sigma$, which is calculated from,

$$
\sigma^{2}=\frac{\epsilon \beta}{-2 \pi \ln (1-F)}
$$

Where $\mathrm{F}$ is the fraction of emittance (e.g., $95 \%$ ). The momentum part of the particle coordinate is calculated using:

$$
\sigma_{\delta}^{2}=\frac{1}{-2 \ln (1-F)}\left(D \frac{\Delta p}{p_{0}}\right)^{2}
$$

Where $D$ is the momentum dispersion of the lattice and $\Delta p / p_{0}$ corresponds to the fraction $\mathrm{F}$ of the total momentum distribution.

$$
\delta=\sigma_{\delta} \sqrt{-2 \ln \left(i_{1}\right)} \cos \left(2 \pi i_{2}\right)
$$

The total beam size depends on both components as:

$$
\sigma_{T}^{2}=\sigma^{2}+\sigma_{\delta}^{2}
$$

Figure 1 shows the result of producing a Gaussian distributed phase space given Courant-Snyder parameters, emittance, and $\Delta p / p_{0}$.

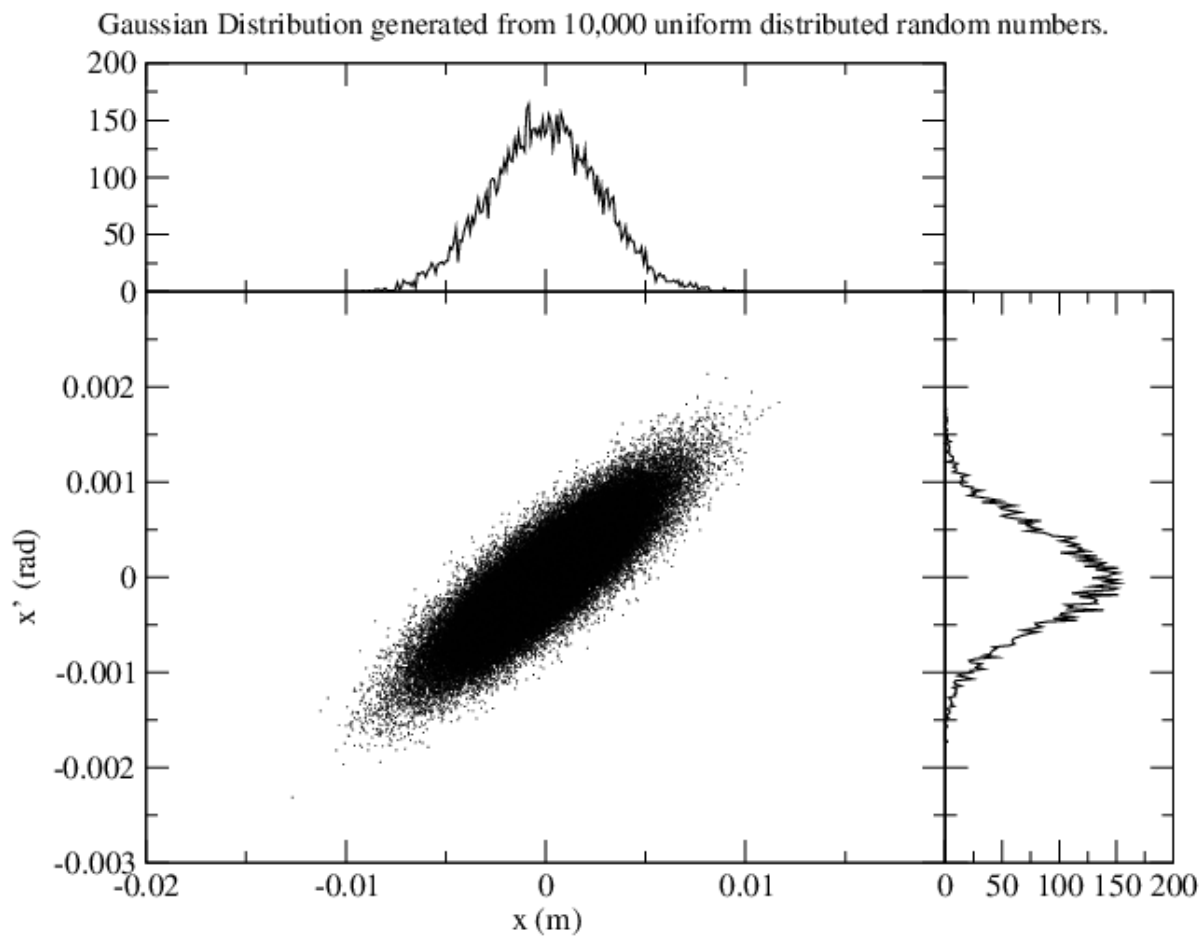

Figure 1: Gaussian generated phase space using uniform random numbers 


\section{TRACKING PARTICLES THROUGH RESONANCES}

If one knows the Courant-Snyder parameters at two points in a lattice, a single particle can be tracked from the first point to the next using the following transformation.

$$
\left(\begin{array}{c}
x_{f} \\
x_{f}^{\prime} \\
\delta_{f}
\end{array}\right)=\left(\begin{array}{lll}
M_{11} & M_{12} & M_{13} \\
M_{21} & M_{22} & M_{23} \\
M_{31} & M_{32} & M_{33}
\end{array}\right)\left(\begin{array}{c}
x_{i} \\
x_{i}^{\prime} \\
\delta_{i}
\end{array}\right)
$$

where

$$
\begin{aligned}
& M_{11}=\left(\frac{\beta_{f}}{\beta_{i}}\right)^{\frac{1}{2}}\left(\cos \Delta \psi+\alpha_{i} \sin \Delta \psi\right) \\
& M_{12}=\left(\beta_{f} \beta_{i}\right)^{\frac{1}{2}} \sin \Delta \psi \\
& M_{13}=D_{f}-\left(\frac{\beta_{f}}{\beta_{i}}\right)^{\frac{1}{2}}\left(\cos \Delta \psi+\alpha_{i} \sin \Delta \psi\right) D_{i}-\left(\beta_{f} \beta_{i}\right)^{\frac{1}{2}} \sin \Delta \psi D_{i}^{\prime} \\
& M_{21}=-\frac{1+\alpha_{i} \alpha_{f}}{\left(\beta_{i} \beta_{f} \frac{1}{2}\right.} \sin \Delta \psi+\frac{\alpha_{i}-\alpha_{f}}{\left(\beta_{i} \beta_{f}\right)^{\frac{1}{2}}} \cos \Delta \psi \\
& M_{22}=\left(\frac{\beta_{i}}{\beta_{f}}\right)^{\frac{1}{2}}\left(\cos \Delta \psi-\alpha_{f} \sin \Delta \psi\right) \\
& M_{23}=D_{f}^{\prime}-\left(-\frac{1+\alpha_{i} \alpha_{f}}{\left(\beta_{i} \beta_{f}\right)^{\frac{1}{2}}} \sin \Delta \psi+\frac{\alpha_{i}-\alpha_{f}}{\left(\beta_{i} \beta_{f}\right)^{\frac{1}{2}}} \cos \Delta \psi\right) D_{i}-\left(\frac{\beta_{i}}{\beta_{f}}\right)^{\frac{1}{2}}\left(\cos \Delta \psi-\alpha_{f} \sin \Delta \psi\right) D_{i}^{\prime} \\
& M_{31}=0 \\
& M_{32}=0 \\
& M_{33}=1
\end{aligned}
$$

where $\delta$ is the momentum deviation for the particle $(\Delta p / p), D_{i, f}$ and $D_{i, f}^{\prime}$ are the dispersion and angular dispersion at the two points. $\alpha_{i, f}$ and $\beta_{i, f}$ are the Courant-Snyder parameters for the two points. The phase advance from $i$ to $f$ is denoted $\Delta \psi$. The vertical plane is evaluated in the same way, although it is assumed there is no coupling terms in between kicks (only coupling produced by the sextupole or octupole kick is included).

As long as there is not a strong momentum dependence of the Courant-Snyder parameters (i.e., a strong radial dependence), then we can assume the values to be static over the range of particles that represent realistic cases. Nevertheless the code has the ability to linearly vary Courant-Snyder parameters if the dependence is specified.

In both the AGS and the Booster a sextupole resonance is created using 2 sets of sextupoles excited in opposite polarities. Therefore, in order to study sextupole resonances in the AGS or Booster we need to define at least 5 points in the lattice: 4 sextupoles and one septum. We can construct the phase space at the septum by putting in thin sextupole kicks (which keeps the transformations symplectic) and tracking particles starting at the septum and observing the particles at the septum every revolution. One then performs the mapping of eq. (27) from the septum location to the first sextupole, using Courant-Snyder parameters derived from MAD, gives a thin sextupole kick, and maps to the next sextupole. The sextupole kick is done by using,

$$
\begin{gathered}
x_{f}^{\prime}=x_{i}^{\prime}+S \cdot\left(x_{i}^{2}+y_{i}^{2}\right) \\
y_{f}^{\prime}=y_{i}^{\prime}-S \cdot\left(2 x_{i} y_{i}\right)
\end{gathered}
$$

where $\mathrm{S}$ is the sextupole kick strength. This strength can be given directly, but is related to the sextupole field gradient by,

$$
S=\frac{1}{2 ! \cdot B \rho} \int_{-\infty}^{\infty} \frac{\partial^{2} B_{r}}{\partial r^{2}} d l
$$

An octupole kick is done by using,

$$
\begin{aligned}
& x_{f}^{\prime}=x_{i}^{\prime}+O \cdot\left(x_{i}^{3}+x_{i} y_{i}^{2}\right) \\
& y_{f}^{\prime}=y_{i}^{\prime}-O \cdot\left(3 x_{i}^{2} y_{i}+y_{i}^{3}\right)
\end{aligned}
$$

where $\mathrm{O}$ is the octupole kick strength. This strength can be given directly, but is related to the octupole field gradient by,

$$
O=\frac{1}{3 ! \cdot B \rho} \int_{-\infty}^{\infty} \frac{\partial^{3} B_{r}}{\partial r^{3}} d l
$$

In the case of chromatic slow extraction, to simulate traversal through a resonance there needs to be the ability to shift the tune (e.g., phase advances). To do this the phase advances, $\Delta \psi$, are all initially shifted up by a small amount, with a scaling sufficient to place all particles above the resonance, in tune, and then decreased a very small amount each revolution, until the particles pass into the resonance and are excited into large amplitude oscillations. 
The scaling is done as,

$$
\text { scale }=\frac{\sqrt{x^{2}+(D \cdot \delta)^{2}}}{\sqrt{-\ln (1-F)\left(\sigma^{2}+\sigma_{\delta}^{2}\right)}}
$$

The phase advances are then shifted as,

$$
\text { phaseshift }=\text { scale } \cdot\left|\xi \frac{\Delta p}{p_{0}}\right|
$$

where $\xi$ is the lattice chromaticity. For these purposes the method is arbitrary. It is computationally faster than other methods, but it is incompatible with defining a time structure to the extracted particles. To do this would slow down the simulations significantly. We need only get enough tune shift to get the particle away from the resonance (since the given Courant-Snyder parameters are for a lattice right at or very close to the resonant tune) and which can be parametrically driven back down into the resonance adiabatically. Figure 2 shows an example of 4 different particles being driven into a sextupole resonance.

In the case of zero chromaticity slow extraction, this approach doesn't work. Instead the tune is shifted by calculating the tune width of the resonance for a given particle amplitude, shifting the phase advances down by that amount, and then stepping the phase advances up (in the case of J-PARC) very slowly. Again this is done for computational efficiency. The tune width is:

$$
\delta Q>\sqrt{x^{2}+x^{\prime 2}} \frac{|S|}{\sqrt{48 \pi \sqrt{3}}}
$$

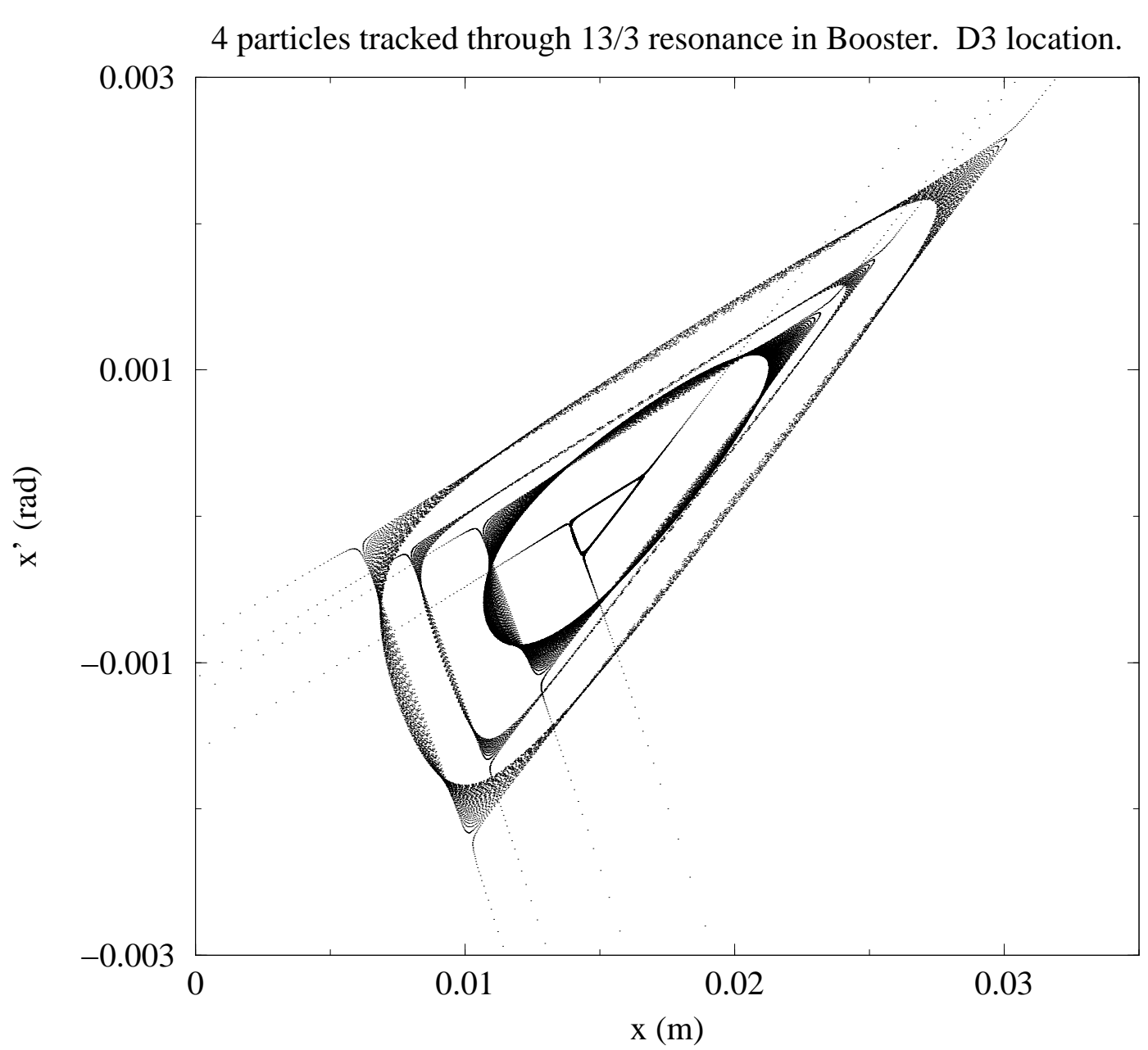

Figure 2: Four particles passing into a sextupole resonance at the Booster D3 thin septum 
Figure 3 is an idealized case showing the $\mathrm{x}$ and $\mathrm{x}$ ' distributions of the horizontal phase space for 10000 particles. Such a distribution is what is to be expected for chromatic slow extraction without any kind of dynamic bump.

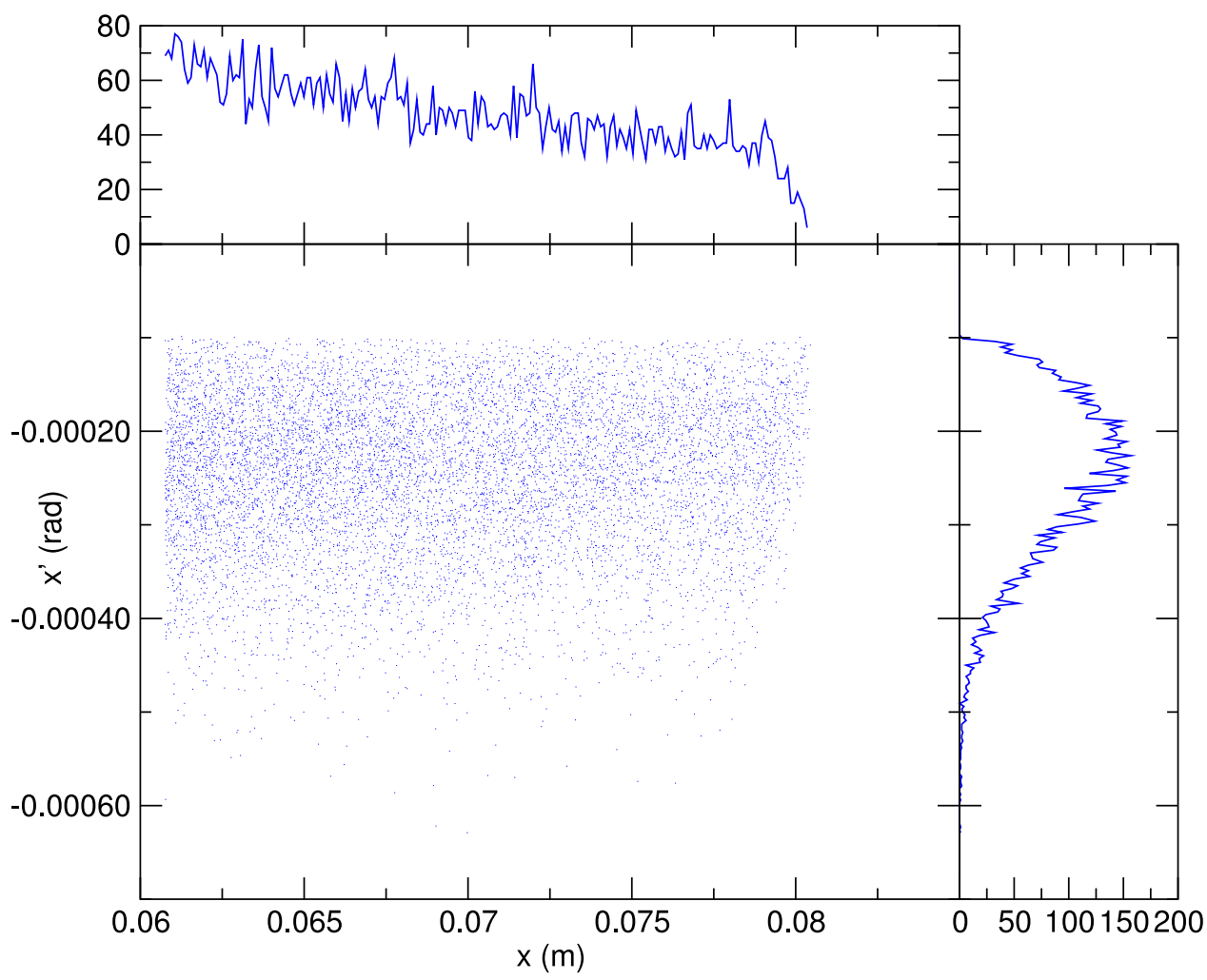

Figure 3: Distribution of particles in $\mathrm{x}$ and $\mathrm{x}$ ' for idealized phase space.

\section{INCLUDING SEPTA AND MEASURING LOSSES}

Defining the septum is relatively simple, since the coordinate system is clearly defined and one only needs to give a location and a thickness. If a particle position falls within the area of septum location + septum thickness, it is considered lost. Scattering from the septum is not considered. This allows calculation of extraction efficiency simply by counting how many particles hit the septum and how many particles fall past the septum. An orbit deviation, or "bump", can be defined to allow matching to other simulations, by using $x$ and $x^{\prime}$ offsets.

Including a dynamic bump is also not difficult, since one only needs to adjust the $x^{\prime}$ offset at the septum according to the initial particle amplitude, as seen in Fig. 5. This dynamic bump keeps the $x^{\prime}$ distribution of the extracted beam minimized, but does not change the step size of the particles at the septum. 


\section{CERN MAD8 SIMULATION OF J-PARC EXTRACTION LATTICE}

Courant-Snyder parameters at the locations of the J-PARC dynamic sextupoles and at the first electrostatic septum (ESS1) were obtained using CERN Mad8 and the J-PARC lattice. The following tables give the parameters obtained from different Mad8 runs. The tracking simulations were performed using the lattice description with a $-35 \mathrm{~mm}$ bump amplitude. Note that the definition of chromaticity in CERN Mad8 is $\xi=\frac{d Q}{d E / p c}$. Table 1 gives the tunes and chromaticities obtained with and without an orbit deformation at the first electrostatic septum. The shift in chromaticity due to the bump is very small. The tracking simulations showed no significant differences with and without this chromaticity. Table 2 gives the Courant-Snyder parameters at the entrance to the ESS1 and at each of the dynamic sextupoles in the J-PARC lattice with the bump on. Table 3 gives the dispersion and orbit parameters at each location. The bump causes a small amount of dispersion at the ESS1.

Table 1: J-PARC Tunes and Chromaticities with and without a bump at ESS1

\begin{tabular}{|l|c|c|c|c|}
\hline Bump Amp(mm) & $Q_{x}$ & $Q_{y}$ & $\xi_{x}$ & $\xi_{y}$ \\
\hline 0 & 22.33333385 & 20.775998 & -0.00495605 & -0.00489664 \\
-35 & 22.33333385 & 20.775998 & -0.276231 & 0.286794 \\
\hline
\end{tabular}

Table 2: Courant-Synder parameters used in tracking simulations.

\begin{tabular}{|l|c|c|c|c|c|c|}
\hline Location Name & $\beta_{x}$ & $\beta_{y}$ & $\mu_{x}$ & $\mu_{y}$ & $\alpha_{x}$ & $\alpha_{y}$ \\
\hline ESS1 & 39.31015260 & 10.19102971 & 0.217285420 & 0.142326351 & 0.019077589 & 0.7171404074 \\
SFMC21 & 11.44370613 & 12.30157391 & 1.849756712 & 1.727043446 & 1.617149793 & -1.740676019 \\
SFMC11 & 11.44211818 & 12.30128657 & 2.599758702 & 2.427046124 & 1.616900394 & -1.740669010 \\
SFMC21 & 11.44370613 & 12.30158476 & 4.849756712 & 4.527046705 & 1.617149793 & -1.740718507 \\
SFMC11 & 11.44211818 & 12.30157391 & 5.599758702 & 5.227043446 & 1.616900394 & -1.740676019 \\
SFMC12 & 11.44211818 & 12.30128657 & 10.04420332 & 9.352378798 & 1.616900394 & -1.740669010 \\
SFMC12 & 11.44211818 & 12.30157391 & 13.04420332 & 12.15237612 & 1.616900394 & -1.740676019 \\
SFMC22 & 11.44370613 & 12.30157391 & 16.73864595 & 15.57770879 & 1.617149793 & -1.740676019 \\
SFMC22 & 11.44370613 & 12.30158476 & 19.73864595 & 18.37771205 & 1.617149793 & -1.740718507 \\
\hline
\end{tabular}

Table 3: Dispersion and orbit deformations.

\begin{tabular}{|l|c|c|c|c|}
\hline Location Name & $D_{x}$ & $D_{x}^{\prime}$ & $x$ & $x^{\prime}$ \\
\hline ESS1 & -0.210942 & $2.08 \mathrm{E}-03$ & -35 & -0.82 \\
SFMC21 & 1.72555 & -0.295192 & $2.29 \mathrm{E}-12$ & $1.07 \mathrm{E}-12$ \\
SFMC11 & 2.22005 & -0.374792 & $-1.60 \mathrm{E}-11$ & $2.46 \mathrm{E}-12$ \\
SFMC21 & 1.72555 & -0.295192 & $2.29 \mathrm{E}-12$ & $1.07 \mathrm{E}-12$ \\
SFMC11 & 2.22005 & -0.374792 & $-1.60 \mathrm{E}-11$ & $2.46 \mathrm{E}-12$ \\
SFMC12 & 2.46185 & -0.427707 & $1.58 \mathrm{E}-11$ & $-1.94 \mathrm{E}-12$ \\
SFMC12 & 2.46185 & -0.427707 & $1.58 \mathrm{E}-11$ & $-1.94 \mathrm{E}-12$ \\
SFMC22 & 1.62991 & -0.27654 & $-8.51 \mathrm{E}-12$ & $2.40 \mathrm{E}-12$ \\
SFMC22 & 1.62991 & -0.27654 & $-8.51 \mathrm{E}-12$ & $2.40 \mathrm{E}-12$ \\
\hline
\end{tabular}




\section{DYNAMIC BUMP FOR J-PARC SLOW EXTRACTION}

The nominal design for the J-PARC slow extraction uses a dynamic bump that minimizes the angular spread of the extracted beam, but does not maintain a constant step size at the septum. The range in positions and angle for this bump, for 3 different particle amplitudes (0,2, and $6 \pi \mathrm{mm}$-mrad (un-normalized)) is shown in figure 4 . The most significant feature is the displacement at SM10 through SM12 increases as the angle is increased at ESS1. I have not looked at the affect of the dymanic bump at these locations in the particle tracking.

It is also possible that the step size at ESS1 can be kept constant, possibly improving the extraction efficiency, by sweeping the position and angle at ESS1. The places to watch, in this case are at SM10 through SM12 and at the inside at SM33. This is a much more complicated bump and would require adding more orbit bump magnets.

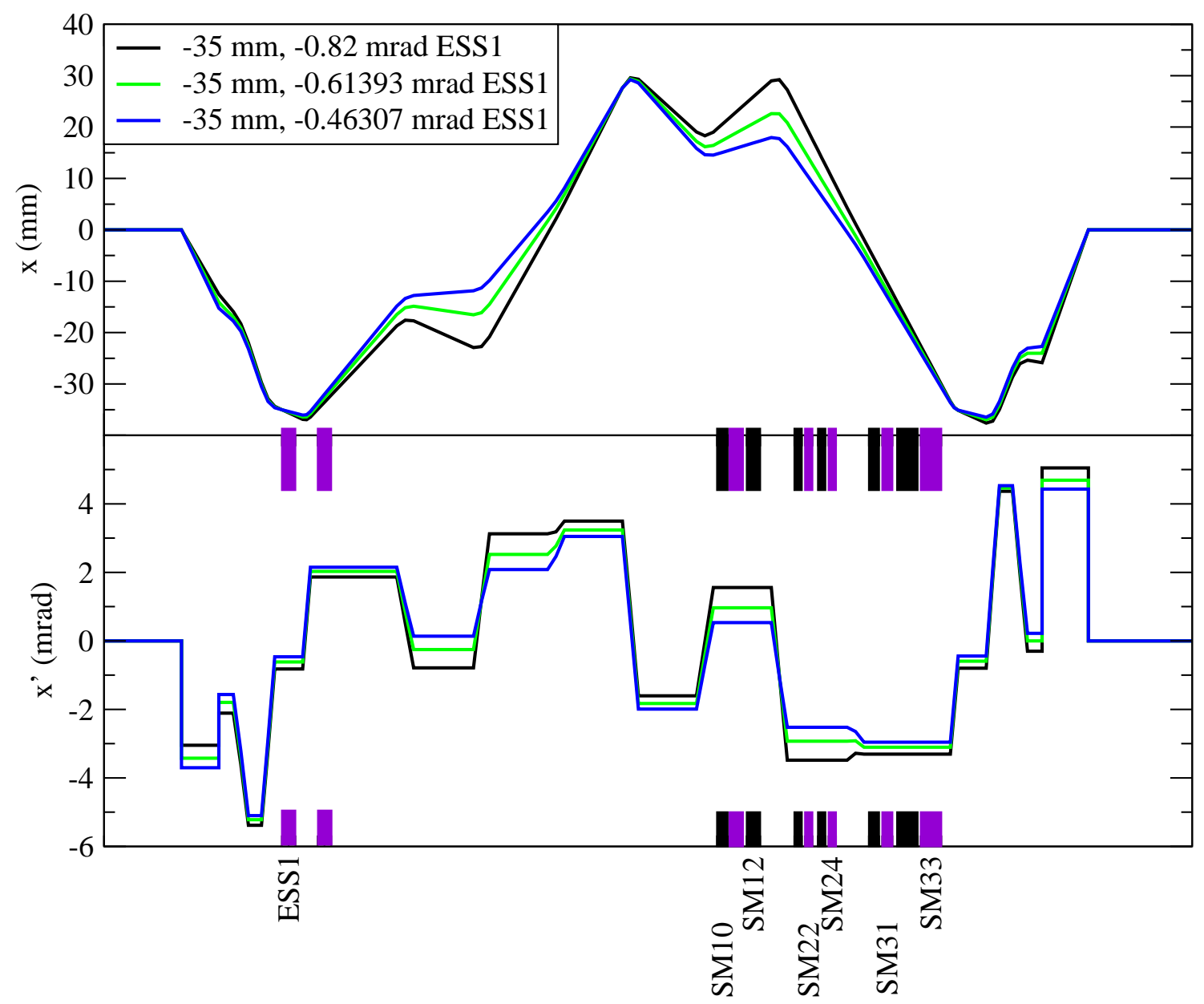

Figure 4: Change in orbit position and angle in extraction region for dynamic bump with constant position and angle that varies from 0 to $-0.25 \mathrm{mrad}$ 


\section{TRACKING SIMULATION OF J-PARC SLOW EXTRACTION}

Figures 5 and 6 show the results of tracking the J-PARC slow extraction system. The extraction efficiency for both cases is $99 \%$. This efficiency is the minimum extraction efficiency, since it assumes that any particle that "hits" the ESS1 is lost. Figure 6 shows a larger angular spread in the particles that have stepped past the septum. The tracking simulation is less reliable for the larger amplitude particles. This is because the tracking simulation uses parameters from a Mad8 simulation and to move particles away from the resonance it is required to shift these parameters. Nevertheless the efficiency is unaffected by this and the extraction process remains the same. The tracking simulation could be improved to better compensate and properly vary lattice parameters, but this would require significant effort and clearly will not offer additional insights to the physics of the process.

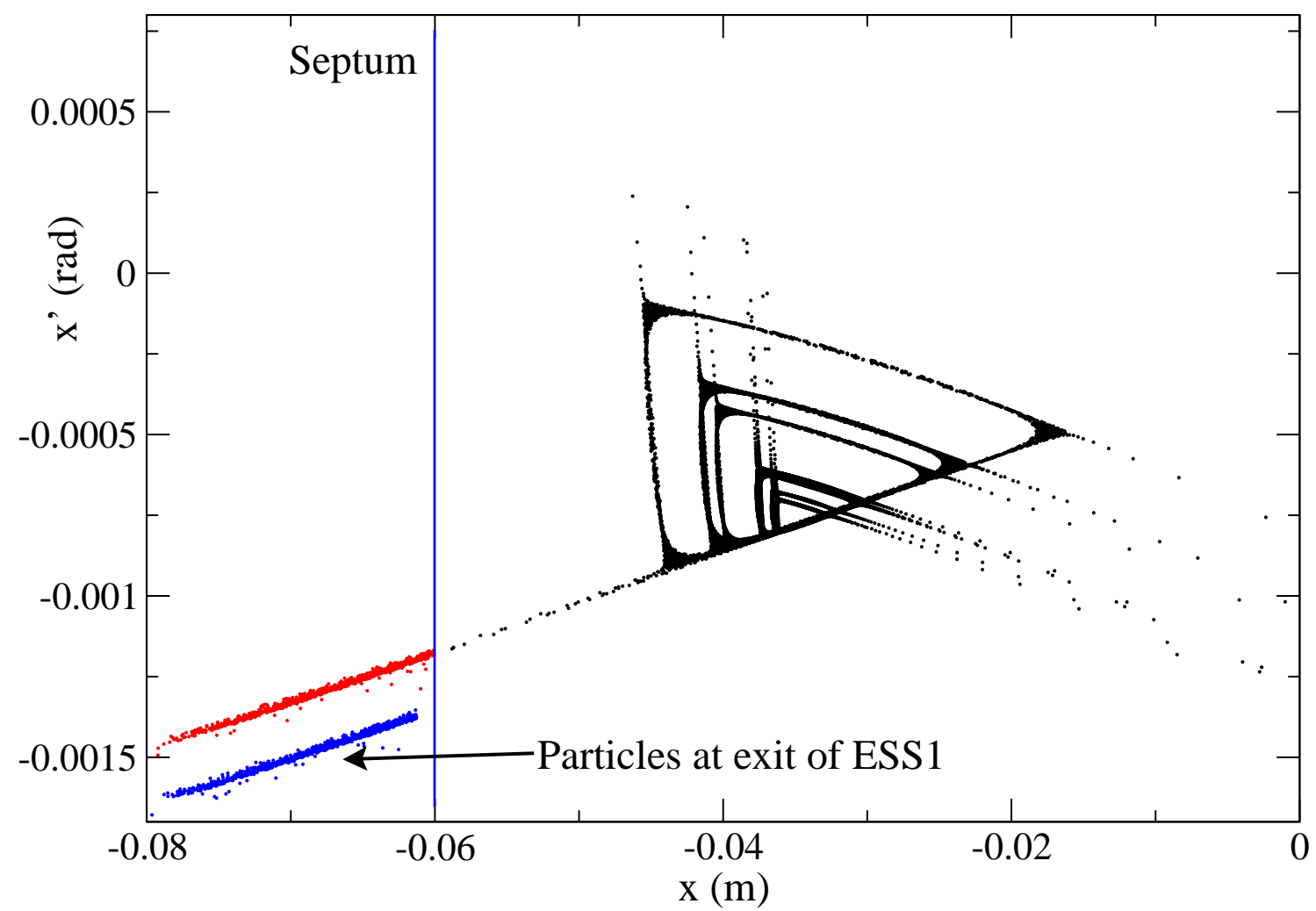

Figure 5: Phase space at J-PARC ESS for 10 different particles (in black), including a dynamic bump. Step size in this example is $2 \mathrm{~cm}$ for small amplitude particles. $>990$ Particles in red have stepped past the septum (blue line at $-0.06 \mathrm{~m}$ ) out of 1000 initial particles. In this case the initial beam emittance is $2 \pi \mathrm{mm}-\mathrm{mrad}$. The septum thickness is taken to be $0.051 \mathrm{~mm}(2 / 1000$ of an inch). Extracted particle phase space trajectories are also shown at the exit of ESS1. 


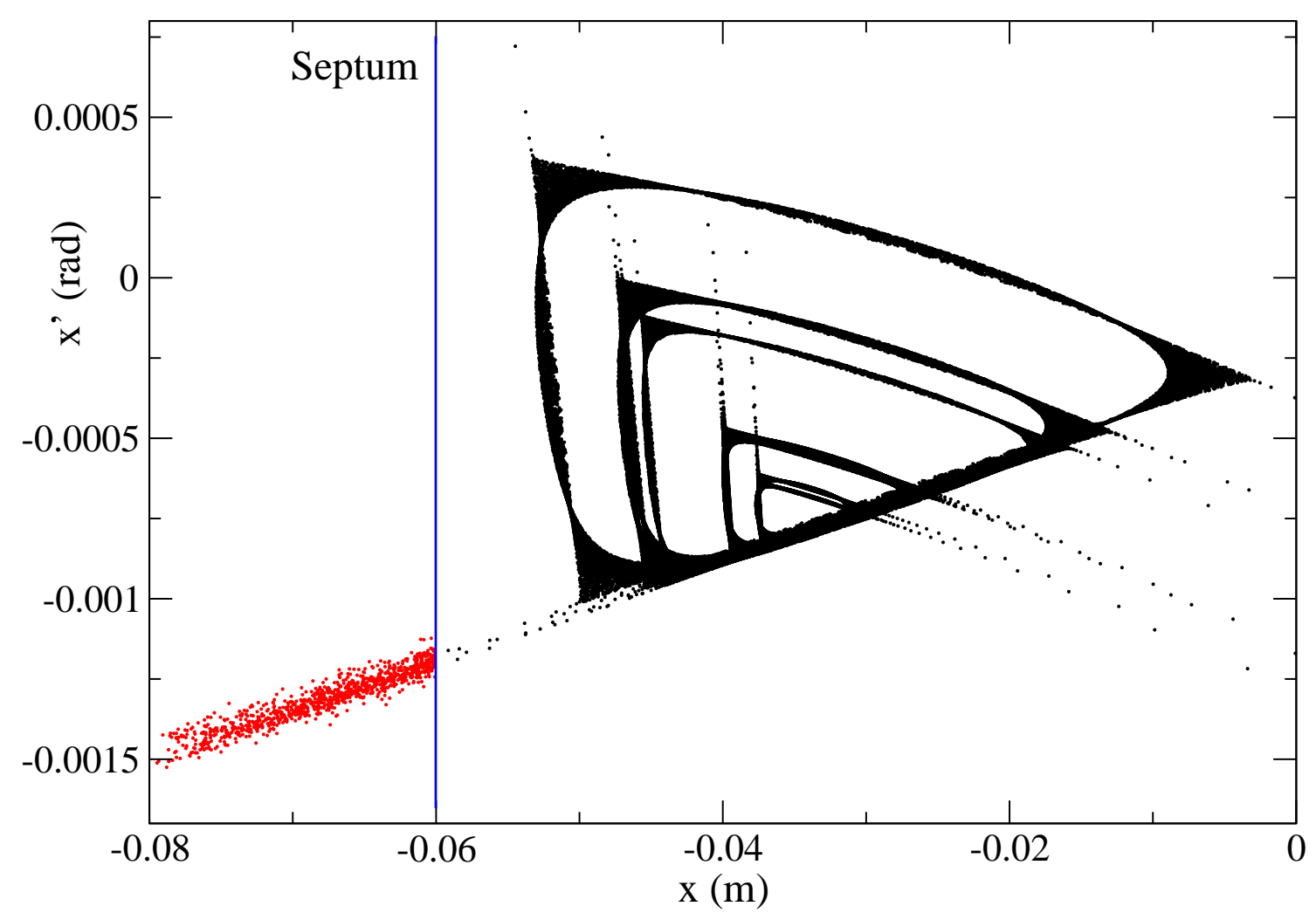

Figure 6: The same conditions as in Fig. 5, except in this case the initial beam emittance is $6 \pi \mathrm{mm}-\mathrm{mrad}$.

\section{AN ALTERNATIVE EXTRACTION SCHEME NOT REQUIRING DYNAMIC BUMPS}

The entire reason for requiring dynamic bumps is because different amplitude particles launch along different trajectories during slow extraction. If all particles could be extracted from the same amplitude, then one would not require a dynamic bump, the angular spread of the beam would be minimized, and the step size for all extracted particles would be fixed. To do this would require performing an "RF knockout" method of slow extraction, in which the particles amplitudes are driven up while keeping the betatron tune fixed. At the high energies of J-PARC this may seem impractical (if not impossible), but one could imagine using an AC-dipole instead of stripline kickers, as normally employed for this method. Since the chromaticity is zero, the AC-dipole would not require frequency modulation (or a very small amount of FM, since chromaticity isn't exactly zero) and it could operate on a subharmonic of the betatron tune, allowing for a lower frequency, and thus lower power, power supply. The AC-dipole, in this case, would need to provide a maximum kick of $1 \mathrm{mrad}$ (or approximately the 99\% 1/2 angular width of the beam at the ESS1). It would adiabatically be ramped up, driving the particles up in amplitude, into the resonance, while not requiring ramping of the tune or the bumps. It could be amplitude or frequency modulated to keep spill structure minimized, using a feedback system similar to that employed in the KEK slow extraction system. Note: since particles are extracted by driving the amplitudes up into the resonance, there will not be large (AC-dipole created) orbit distortions in other locations of the J-PARC lattice, and so no additional aperture is used when this method of extraction is employed.

\section{CONCLUSIONS}

The J-PARC slow extraction system is a unique and elegent design. The simulations done here show that this system will work as expected. Not all questions relating to slow extraction from J-PARC have been addressed, though. I believe achieving an extraction efficiency of $99 \%$ or better will be achievable given this design. Further improvements in the design, such as the idea of using a pre-scatter septum, have not been studied here, but this seems to be a very reasonable method of reducing activation on extraction equipment. It is an idea worth pursuing in significant detail. Another method of achieving higher efficiency is to explore the use higher order multipoles in the extraction system. This is also an area worth exploring in some detail.

Effects due to high intensity operation have not been explored in this report. Some area's worth exploring in more detail are: 
1. Compensation for coherent space charge tune shift during extraction.

2. Incoherent space charge will reduce the dynamic aperture initially available for slow extraction. How significant is this?

3. The beam being extracted has to move from the inside of the accelerator aperture to the outside of the accelerator aperture, necessarily having to cross the circulating beam. At high intensity will the two beams have any affect on each other? What is the degree of scattering due to the crossing?

\section{ACKNOWLEDGEMENTS}

E.D.Courant, BNL, was extremely helpful by explaining how to correctly include dispersion in the matrix transformations. The J-PARC lattice was obtained from M. Tomizawa, KEK.

\section{REFERENCES}

[1] D.Edwards and M.Syphers, "An Introduction to the Physics of High Energy Accelerators", Wiley Series in Beam Physics, 1993 John Wiley \& Sons, Inc. ISBN 0-471-55163-5

[2] H.Grote, F.C.Iselin, “The MAD Program User's Reference Manual”, CERN/SL/90-13(AP)Rev.4, May 26, 1995. 